\title{
Preliminary Experience with Rifampicin and Isoprodian in Combination in Leprosy Treatment
}

\author{
H. N. KRENZIEN \\ Balaka Leprosy Hospital, Malawi
}

\begin{abstract}
Sixty-seven patients with lepromatous leprosy were given combined treatment with rifampicin and Isoprodian at Balaka Leprosy Hospital in Malawi, and experience during the first 15 months is described. Administration was orally according to body weight. Several criteria of control were applied. In addition to routine skin smears, serial biopsies were taken simultaneously, homogenized, the bacilli counted and the bacillary load of the skin calculated per $\mathrm{mg}$ of tissue. Clinical improvement from moderate to dramatic occurred in all patients in a matter of months. The fall of the BI was on average 1 unit on Ridley's scale, the homogenate counts indicate a bacilli reduction of more than $90 \%$ after 1 year of treatment. A comparison of the simultaneously taken skin smears and biopsy counts is undertaken. The frequency of reactional states under combined therapy and the relationship to secondary parasitic infectious diseases is described. Side-effects were mostly transitory, in 5 cases the combination tablet Isoprodian was discontinued. Some patients showed slightly elevated liver enzymes. However, more biochemical investigation is needed with regard to liver and kidney function under this therapy.
\end{abstract}

Since June, 1973 a combined therapy which has been developed on an experimental basis at the Forschungsinstitut Borstel has been applied at Balaka Leprosy Hospital in Malawi to patients with lepromatous leprosy.

\section{Drugs, Application, Dosage}

The antimycobacterial effective drugs of this combination are rifampicin, isonazid, prothionamide and dapsone. The drugs were given orally after breakfast in the morning in one dosage according to $\mathrm{kg} /$ bodyweight, rifampicin in the dosage of $10 \mathrm{mg}$, isoniazid and prothionamide $5 \mathrm{mg}$ and dapsone 2 per $\mathrm{kg}$ bodyweight. The average patient with a bodyweight of $60 \mathrm{~kg}$ received accordingly 2 capsules rifampicin, $300 \mathrm{mg}$ each, and 2 tablets of Isoprodian, the combined tablet of INH, PTH and DDS. Medications were given daily except on Sundays to ensure the elimination of the drugs or their metabolic products.

\section{Criteria of Control}

Several criteria of control have been used. Besides the clinical examination, serial photos have been taken at intervals to document progress. Before the start of therapy and during therapy routine skin smears and small biopsies have been taken at monthly intervals. The biopsies are used to quantitative determination of 
the bacilli in the skin and for histological examination.

The amount of bacilli in the skin biopsies is counted with the same method which is used by Shepard and Rees in their mouse footpad experiments. After taking the biopsy the material is fixed in $4 \%$ formalin and later divided into 2 parts. One is to be used for cryo-sections, the other one is homogenized in Belco tissue grinders for the bacilli count. Before starting homogenizing, the biopsy is weighed on an analytic scale, the average weight of the skin biopsies varies between 15-40 mg. The tissue is then homogenized in the volume of $1 \mathrm{ml} 0.1 \%$ albumin/dist. water in tissue grinders. Having reached a fine suspension $5 \mu 1$ of the homogenate are equally distributed on spot-slides. The slides are air-dried, fixed on a heating plate, Ziehl-Neelsen stained and the bacilli counted under high power. Finally the amount of bacilli is calculated per $\mathrm{mg} /$ tissue.

\section{Section of Patients}

From June 1973 to August 1974, 67 lepromatous patients have been taken under the combined therapy and by the end of this year 100 patients will be included. Forty-eight cases had been previously treated with dapsone 19 were new, previously untreated patients. In 13 patients the duration of previous treatment lasted up to 5 years, the majority of 35 cases had been previously for between 5 and 25 years with dapsone on different therapy schedules. During the last 7-8 years most of them had $25 \mathrm{mg}$ DDS daily as this schedule was used throughout the country of Malawi since 1965. One section of the patients had

\section{TABLE 1}

Distribution of patients as between untreated and previously treated groups

\begin{tabular}{|c|c|c|}
\hline \multicolumn{2}{|c|}{ Total patients } & 67 \\
\hline \multicolumn{2}{|c|}{ Previously untreated } & 19 \\
\hline \multicolumn{2}{|c|}{ Treated previously } & \\
\hline $\begin{array}{l}\text { No. of years } \\
0-5 \\
6-10 \\
11-15 \\
16-20 \\
21-25\end{array}$ & $\begin{array}{c}\text { No. of patients } \\
13 \\
14 \\
8 \\
5 \\
8\end{array}$ & \\
\hline $21-25$ & Total & 48 \\
\hline
\end{tabular}

TABLE 2

Age-and sex-distribution of 67 patients taken under combined therapy. All had lepromatous leprosy

\begin{tabular}{ccc}
\hline Total patients & Age groups & No. of patients \\
\hline 67 & $0-14$ years & 3 \\
$\left(520^{\circ}\right.$ & $15-39$ years & 33 \\
$159)$ & $40-59$ years & 30 \\
& 60 and over & 1 \\
\hline
\end{tabular}


been hospitalized for several years in different leprosaria, which ensured regular treatment to a certain degree. However, irregularity in taking the previous treatment certainly must be considered, although the majority of the cases previously treated but unregistered claimed to have taken their dapsone regularly over all the years as out-patients.

The age- and sex-distribution of the patients was as follows: 52 out of 67 patients are male, 15 patients are female. Three children with lepromatous are within the age-group up to 14 years, the majority are in the age-groups 15-39 and 40-59 years. One patient was more than 60 years old at the beginning of therapy.

\section{Clinical Results}

In all 67 cases a clinical improvement was seen not only by the examiner but by the patients themselves. This seems to me very important and one reason for the good attendance at the out-patient clinics. The time and degree of the clinical improvement varied from the dramatic within a short time of 1 to 3 months to slow clinical improvement within 12 months and longer.

The reddish succulent papules and nodules from new, previously untreated cases, and from obviously relapsed cases disappeared early, whereas the subsidence of old indurative lesions took longer, at least 12 months or more. There are examples of an impressive dramatic clinical improvement in cases treated previously with dapsone for many years whose physiognomy changed from week to week to the better, all papules on the body subsided within three months; these patients never came into reactional states. (The author demonstrated numerous photos comparing the patients before and after treatment.)

\section{Bacteriological Results}

The skin smears of most patients gave a Bacteriological Index of between 4+ and 5+ on Ridley's logarithmic scale before therapy.

Figure 1 gives the mean results of skin smears at 3-monthly intervals, the figures in brackets giving the number of patients. The figure for the first quarter

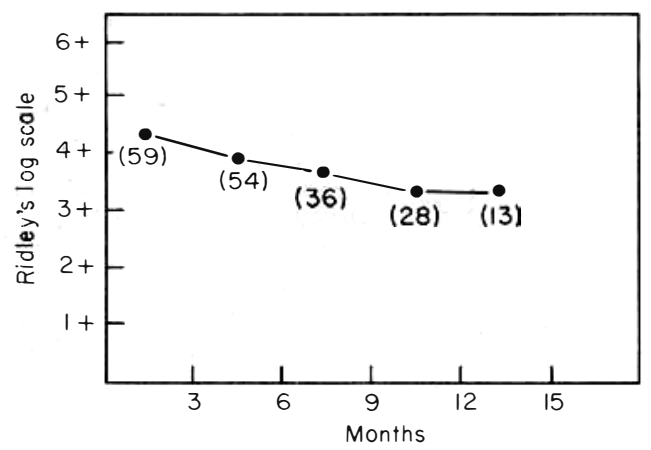

Fig. 1. Decrease of BI with combined therapy. Mean skin smears before combined therapy and up to 15 months after. The figures in brackets indicate the number of patients investigated. The average fall of the BI was 1 unit of the Ridley scale within 12 months. 
of the year includes the mean of the figure at the start and those after 1 and 2 months, so that the total of skin smears taken into consideration amounts to $59 \times 3=177$ smears. The figure shows the slow decrease of the BI after 12-15 months of combined therapy. The BI had gone down on average 1 unit of Ridley's scale, in this case from $4.4+$ to $3.4+$.

In Fig. 2 the bacilli counts are plotted against time after treatment. The first 3 counts of the first quarter were chosen as starting point $=100 \%$. The figures in brackets again give the number of patients on whom the results are based. The biopsy counts demonstrate a rapid decrease within the first 12 months of therapy, which means an elimination of more than $90 \%$ of the bacillary load of the skin within the first year.

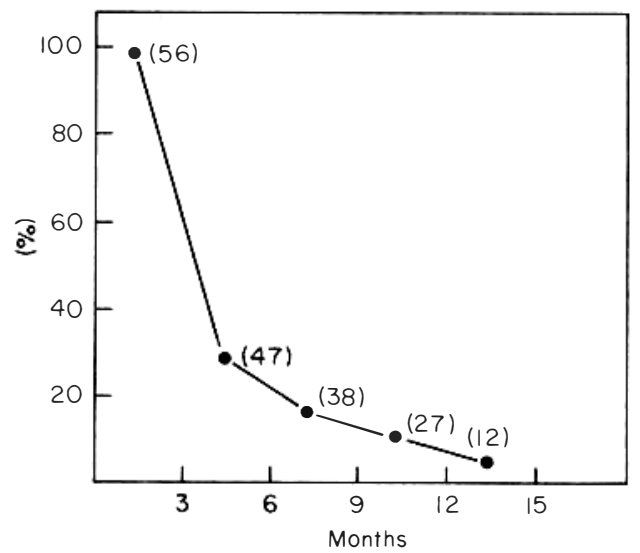

Fig. 2. Decrease of homogenate counts after treatment with rifampicin and Isoprodian. The averages of biopsy count No. 1-3, 4-6, 7-9, 10-12 and 13-15 are plotted against months of treatment. After 12 months of treatment the counts indicate an elimination of more than $90 \%$ of the bacilli load before therapy.

Table 3 shows the actual figures of all homogenate counts before therapy, at

TABLE 3

Elimination of Myco. leprae with rifampicin and Isoprodian

\begin{tabular}{|c|c|c|c|c|}
\hline Time & \multicolumn{2}{|c|}{ Count/bacilli/mg tissue } & $\%$ left & Reduction rate \\
\hline \multicolumn{5}{|c|}{ Before treatment } \\
\hline & $4.94 \times 10^{5}$ & $(64)$ & 100 & 0 \\
\hline \multicolumn{5}{|c|}{ After months of treatment } \\
\hline $\begin{array}{r}1 \\
3 \\
6 \\
9 \\
12\end{array}$ & $\begin{array}{l}3.24 \times 10^{5} \\
1.31 \times 10^{5} \\
6.27 \times 10^{4} \\
3.03 \times 10^{4} \\
9.29 \times 10^{3}\end{array}$ & $\begin{array}{r}(60) \\
(51) \\
(32) \\
(22) \\
(9)\end{array}$ & $\begin{array}{r}65.6 \\
26.5 \\
12.7 \\
6.1 \\
1.9\end{array}$ & $\begin{array}{l}34.4 \\
73.5 \\
87.3 \\
93.9 \\
98.1\end{array}$ \\
\hline
\end{tabular}

The actual figures of the biopsy counts before and after $x$ months of treatment. The figures in brackets give the number of patients upon which the mean counts are based. 
the end of the 1st, 3rd, 6th, 9th and 12 th month of the year. In brackets the number of patients investigated, next to that the percentage of the remaining amount of bacilli and the reduction rate. At the end of the 12 th month a reduction rate of $98 \%$ is reached on average, which means that only $2 \%$ of the bacillary load before therapy is left.

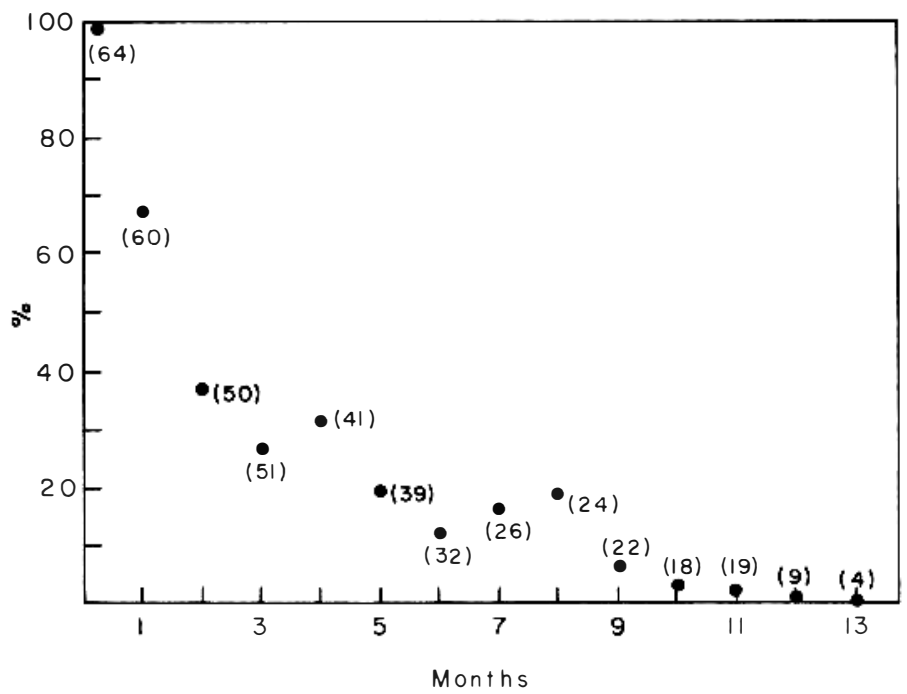

Fig. 3. Elimination of Myco. leprae with rifampicin and Isoprodian. Homogenate counts before therapy and thereafter in monthly intervals plotted against time. Figures in brackets indicate the number of patients investigated.

In Fig. 3 the means of all monthly homogenate counts are plotted against time. The graph indicates that the elimination of bacilli follows the shape of a hyperbola, the elimination of $50 \%$ of the bacilli being already reached between the 1 st and 2 nd month after the therapy. After 10 months of therapy all mean counts fall below the $10 \%$ mark compared with counts before therapy. No

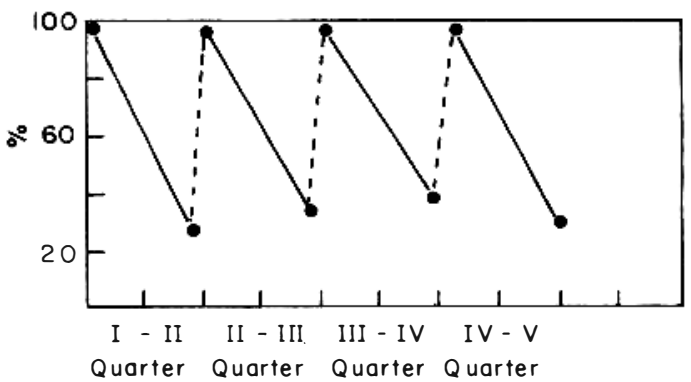

Fig. 4. Bacilli-reduction (relative \%), quarterly comparison. A quarterly comparison shows that the reduction rate between 2 quarters of treatment amounts always $60-70 \%$, when the count of the preceding quarter is brought again to $100 \%$. 
difference in the reduction rates could be seen between previously treated and new previously untreated cases.

Fig. 4 shows the average in percentage of the homogenate counts from all patients in quarterly comparisons. The means of the first 3 biopsy counts are compared with the corresponding figures of the 2 nd quarter, the result of the 2 nd quarter with that of the $3 \mathrm{rd}$ quarter etc., hereby is the preceding mean count always brought to $100 \%$.

Remarkable is that the reduction rates are all within the same range between $60-70 \%$.

As the data of a large number of biopsy counts and simultaneously taken skin smears were available all counts were compared with corresponding skin smears.

Table 4 gives the information about the actual number of bacilli belonging to a result on Ridley's log scale. The figures in brackets give you the amount of investigations upon which the results are based. Ridley's $2+$ to $2.9+$ means that you can expect an average of $6 \times 10^{3}$ bacilli per mg of skin tissue, $3+$ to $4+$ corresponds to $9 \times 10^{3}$ up to $9 \times 10^{4}$ bacilli per mg of tissue. From $4+$ to $5+$ only an increase of half a $\log$, i.e. from $5+$ upwards only 20 comparable results were available, the corresponding figure is $6.7 \times 10^{5} \mathrm{bacilli} / \mathrm{mg}$ of tissue.

TABLE 4

Quantitative comparison

\begin{tabular}{ccc}
\hline $\begin{array}{l}\text { Routine skin smear } \\
\text { (Ridley's log scale) }\end{array}$ & $\begin{array}{c}\text { Biopsy counts } \\
\text { (No. bacilli/mg) }\end{array}$ & $\begin{array}{c}\text { No. of } \\
\text { patients }\end{array}$ \\
\hline $2-2.9$ & $6.48 \times 10^{3}$ & $(36)$ \\
$3-3.5$ & $9.15 \times 10^{3}$ & $(50)$ \\
$3.6-4$ & $9.20 \times 10^{4}$ & $(96)$ \\
$4.1-4.5$ & $1.82 \times 10^{5}$ & $(134)$ \\
$4.6-5$ & $4.88 \times 10^{5}$ & $(56)$ \\
$5.1-5.5$ & $6.72 \times 10^{5}$ & $(20)$ \\
\hline
\end{tabular}

On the left side the figures of Ridley's log scale from $2+$ to $5.5+$. The right side shows the corresponding biopsy counts with the actual number of bacilli/mg tissue. The figures in brackets give the number of patients investigated.

\section{Morphological Indices}

The Morphological Index was followed up after medication with the combined therapy in 31 patients in whom skin smears were taken weekly. The white symbols on Fig. 5 show Morphological Indices before therapy, the black spots any positive result after the beginning of the combined therapy. The MIs were distributed between 1 and $25 \%$ before therapy. The decrease to zero in the MI took place within 12 weeks in all patients. In reading the MIs only solid and unsolid forms were differentiated. The weekly follow-up showed clearly that there is a relationship between the height of the MI and the length of time until negativity. In this respect, no difference in morphological response was found between previously treated and new, previously untreated patients. 


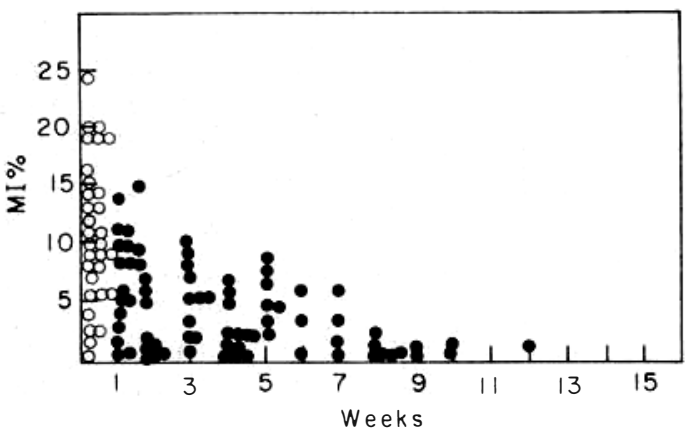

Fig. 5. Decrease of MI with combined therapy rifampicin and Isoprodian. The weekly follow-up of the MI after combined therapy. Each white circle (on the slide red) gives the percentage of solids before therapy, the black circles any positive MI after therapy. The last positive MI is seen after 12 weeks.

\section{Reactional States}

The frequency of reactional states is shown in Fig. 6. Out of 67 patients, 23 showed reactional states during the observation period of 15 months. This includes those patients who were admitted because of reactions and then treated with the combined therapy. The type of reaction varied between pure neuritis, reaction type I and reaction type II. In 4 cases isolated symptoms of neuritis dominated, 2 cases with reaction type I and the remaining 17 were classified as reaction type II with ENL. Out of these 19, 7 reactions occurred in new, previously untreated cases and 12 in previously treated cases, most of whom had had several reactions previously.

\section{Secondary parasitic infections}

Fifty-eight out of 67 patients were started on the combined therapy in the hospital after being checked for diseases other than leprosy. This included in all cases urine and stool analysis as routine examination for parasitic infections.

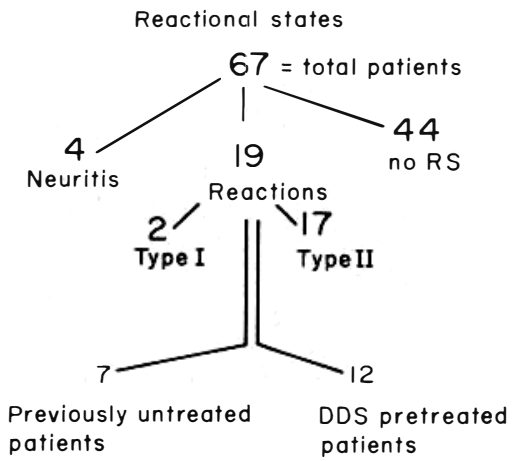

Fig. 6 . The frequency of reactional states among the 67 lepromatous patients. 


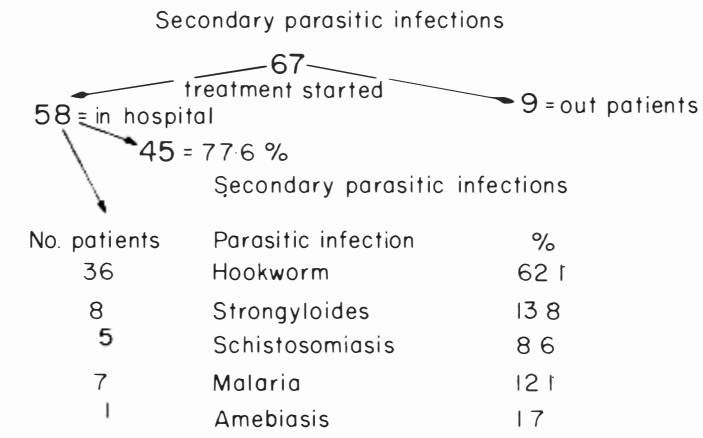

Fig. 7. The frequency of secondary parasitic infectious diseases checked during the first weeks when admitted in the hospital.

Forty-five out of 58 cases $(77.6 \%)$ showed secondary parasitic infections. The incidence of the different parasitic infections is given in Fig. 7; the frequency of parasitic infections among those 19 patients with reactional states is shown in Table 5.

Four patients were free from parasitic infection, 15 patients had at least 1 or even 3 parasitic infectious diseases at the same time besides lepromatous leprosy.

TABLE 5

The relationship between patients with reactional states and their possible reason-the secondary infectious diseases

\begin{tabular}{cc}
\hline $\begin{array}{c}\text { Reactions-secondary parasitic infections } \\
\text { No. patients }\end{array}$ & No. sec. par. inf. \\
\hline 4 & 0 \\
7 & 1 \\
5 & 2 \\
3 & 3 \\
\hline
\end{tabular}

\section{Side-effects}

As the equipment for biochemical investigations arrived only recently, liver and kidney function tests on patients under combined therapy are still in the early stages. So far 17 patients under combined therapy have been tested for SGOT and SGPT levels. Of these 9 showed slightly elevated results in SGOT and 6 moderate elevations in SGPT after different length of therapy. Alkaline phosphatase was determined in 14 patients after different periods of therapy and in 3 cases slight elevation was found. Although the number of investigations is very limited and no test before the beginning of therapy had been possible, this could be an indicator for hepatotoxic reaction and further investigations are necessary. The actual figures for the enzyme activities are shown in Figs 8 and 9. 


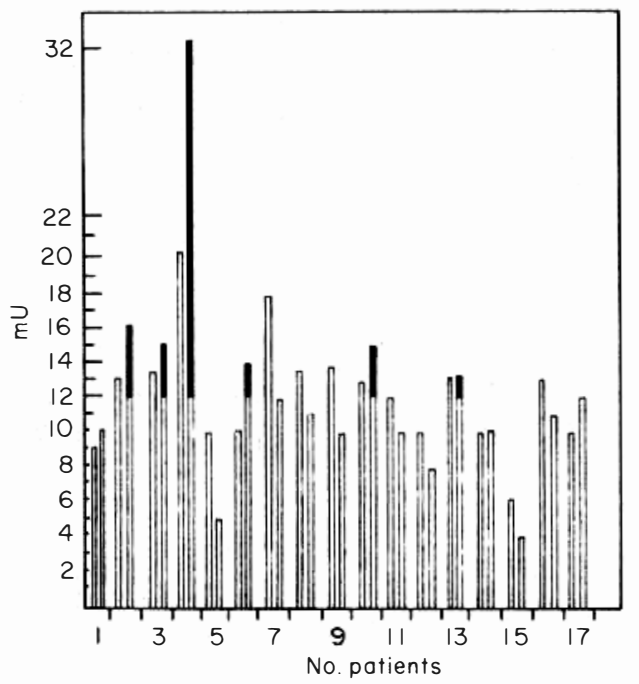

Fig. 8. Enzyme activity under combined therapy (Merkotest Micro-method). SGOT and SGPT investigated in 17 patients after different lengths of treatment with rifampicin and Isoprodian.

$\square=$ Elevated SGOT; $\boldsymbol{m}=$ Elevated SGPT.

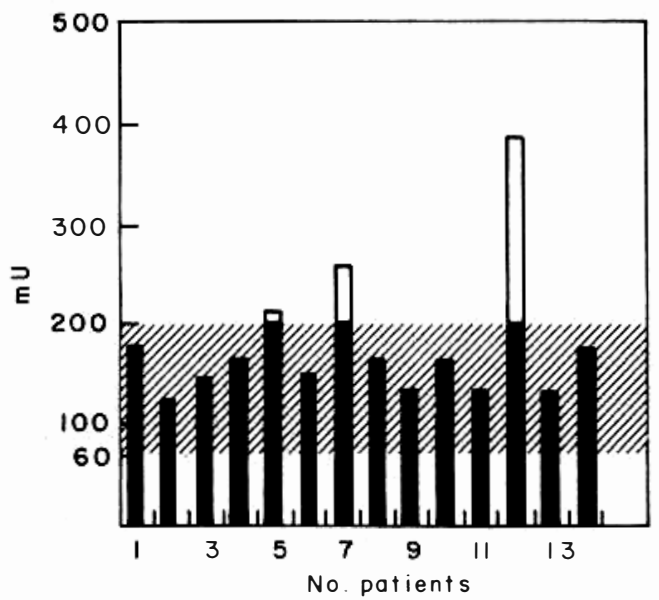

Fig. 9. Enzyme activity under combined therapy (Merckotest Micro-method). The alkaline phosphatase slightly elevated in 3 patients out of 14 investigated after different lengths of treatment with rifampicin and Isoprodian.

$\square=$ Elevated alkaline phosphatase. 
Clinical side-effects were in most cases transitory. Complaints differed from abdominal discomfort, stomach ache, loss of appetite and constipation to headache. Nausea, vomiting and heart palpitations necessitating in 5 cases the withdrawal of Isoprodian. All subjective side-effects stopped after that procedure, so it seems very likely that the abdominal side-effects were due to the prothionamide in the combination tablet Isoprodian.

\section{Acknowledgements}

For advice and support I am grateful to Prof. Dr Dr E. Freerksen, Forschungsinstitut Borstel, and Deutsches Aussaetzigen Hilfswerk, Wuerzburg; I owe thanks to my wife for the extensive laboratory work needed for this investigation. 